\title{
Healthy Infant Born to a Mother Infected with Coronavirus Disease 2019 (COVID-19): A Case Report from Lebanon
} \author{
Chami, $R N^{3}$, Nada Ghosn, MD ${ }^{1}$ and Hamad Hassan, PhD ${ }^{4}$ \\ ${ }^{1}$ Epidemiological Surveillance Program, Ministry of Public Health, Beirut, Lebanon \\ ${ }^{2}$ Department of Obstetrician and Gynecologist, Monla Hospital, Lebanon \\ ${ }^{3}$ Department of Infectious Disease, Monla Hospital, Tripoli, Lebanon \\ ${ }^{4}$ Ministry of Public Health, Beirut, Lebanon
}

Linda Abou-Abbas, PhD ${ }^{1}$ D, Zeina Nasser, PhD $D^{1}$, Abdelwahab El Aly, MD², Matta Matta, MD ${ }^{3}$, Inas El

\begin{abstract}
Objectives: This case report describes the clinical outcomes of pregnant women infected with Coronavirus Disease 2019 (COVID-19) and assesses the vertical transmission of SARS-CoV-2 infection from the pregnant women to the neonate.

Case presentation: A 37-year-old pregnant woman with COVID-19 infection presented to the hospital to deliver her baby. With careful monitoring, the patient underwent an uneventful pregnancy and delivered a healthy full-term infant. There was no potential vertical transmission of the virus to the infant. Breastfeeding appears possible and safe when all appropriate precautions to avoid COVID-19 transmission to the infant are considered.

Conclusion: We reported a successful experience of safe vaginal delivery in a woman with COVID-19. The preparedness of hospitals and healthcare staff for the adequate implementation of infection control practice is crucial for safe delivery. Education of pregnant women about prevention of COVID-19 remains important strategy for protecting maternal and infant health.
\end{abstract}

Keywords

COVID-19, Pregnant Women, Healthy Infant, Lebanon, Case report

\section{Background}

A novel severe acute respiratory syndrome coronavirus 2 (SARS-CoV-2) was first emerged in Wuhan, China in December 2019 and rapidly became an international public health emergency [1,2]. Pregnant women represent a unique challenge during this pandemic. The most important inquiry to the mother and the obstetric team is whether the COVID-19 could be transmitted vertically to the fetus during pregnancy. So far, data have been limited, and the potential risk of vertical transmission is unclear. This case report describes the clinical outcomes of the first case of a COVID-19 pregnant woman in Lebanon who presented to the hospital to deliver her infant and assesses the vertical transmission potential of SARS-CoV-2 infection from the pregnant women to the neonate.

\section{Case Presentation}

On March 18, 2020, early in the morning, a 37-year-old pregnant at 35 weeks of gestation, presented to the labor and delivery unit with symptoms of term labor including increased pressure in pelvis, cramping in lower abdomen, increased vaginal discharge, amniotic fluid leaking from the vagina, and light vaginal bleeding. Upon arrival to the hospital, in the triage room, her husband's temperature was $38.5^{\circ} \mathrm{C}$ and reported history of three days fever, dry cough and sneezing. After considering the husband as suspected COVID-19 case, he was immediately separated from his wife and referred to a reference laboratory where a nasopharyngeal swab for Reverse transcription polymerase chain reaction (RT-PCR) testing was collected. The hospital refused to admit his wife. She was given a surgical mask and she went on the same day at $7 \mathrm{AM}$ to another hospital where infection control

*Corresponding author: Dr. Linda Abou Abbas, Epidemiological Surveillance Program, Ministry of Public Health, Beirut, Lebanon

Accepted: December 29, 2020

Published online: December 31, 2020

Citation: Abou-Abbas L, Nasser Z, Aly A, et al. (2020) Healthy Infant Born to a Mother Infected with Coronavirus Disease 2019 (COVID-19): A Case Report from Lebanon. J Nurs Pract 3(1):221223

Copyright: (C) 2020 Abou-Abbas L, et al. This is an open-access article distributed under the terms of the Creative Commons Attribution License, which permits unrestricted use, distribution, and reproduction in any medium, provided the original author and source are credited. 
precautions measures were taken. Upon admission, the patient's temperature was $36.6{ }^{\circ} \mathrm{C}$, and the blood pressure $133 / 75 \mathrm{mmHg}$. No suspicious symptoms of COVID-19 were remarked. During the labor period, she was afebrile and her vital signs were stable with blood oxygen saturation of $99 \%$. At 6:30 PM she delivered her infant without any complication. The neonate was examined by a pediatric who reported that she is healthy with a birth weight of $3,000 \mathrm{~g}$. The mother and her neonate were discharged home on March 19, 2020 at 11 AM.

On the same day, her husband test result turned positive. Consequently, she was urged by the Ministry of Public Health (MOPH) to do the test together with her infant. On March 20, 2020, a nasopharyngeal swab was collected from her and she got positive result for COVID-19 RT-PCR test. After 72 hours, a throat swab was collected from the infant and the result was negative. An investigation was immediately initiated by the MOPH to identify the demographic characteristics, symptoms, source of infection and underlying conditions. In addition, daily call follow-up for 3 weeks was conducted. Concerning symptoms, the mother reported only the loss of the smell sense few days after delivery. The MOPH found out that the source of her infection was her husband. No underlying conditions were recorded. She was advised by the $\mathrm{MOPH}$ and her gynecologist to breastfeed her infant. She was also provided by the appropriate recommendations on safe feeding and measures to prevent SARS-CoV-2 transmission to her neonate. The mother kept on breastfeeding for 6 weeks after delivery. During this period, she was home isolated; she practiced physical distancing (1.5 meters away from her baby). She didn't practice skin-to-skin contact with her infant. Before each feeding, she kept on washing hands and wearing face mask. RT-PCR was repeated for the infant after 2 weeks of birth and the result was negative. On April 9, 2020, Chest $X$-ray for the mother indicated absence of progressive pleuroparenchymal focus. On $13^{\text {th }}$ and $17^{\text {th }}$ of April 2020, the mother had two negative consecutives RT-PCR.

It is worth mentioning that the mother consulted a psychiatrist to seek psychological support after black thoughts and panic attack she suffered from. She was diagnosed having anxiety due to her experience of COVID-19. As a consequence, she stopped breastfeeding after six weeks.

On the basis of contact tracing, 16 health care workers and the patient's roommate were identified as having direct or indirect contact with the patient at the hospital. Thus, all health care workers were followed up to 14 days with daily monitoring for COVID-19 symptoms by the infection control unit of the hospital. During this period, none of them developed the symptoms. Moreover, upon admission and before knowing the PCR result, the patient had a 40 year pregnant woman roommate who was admitted to the hospital on $18^{\text {th }}$ of April 2020 and discharged next day. The latter has been home quarantined for a period of 14 days from her last exposure to patient with daily monitoring for COVID-19 symptoms by the MOPH. A nasopharyngeal swab for SARS-CoV-2 PCR test was collected and the result was negative.

\section{Discussion}

This case describes uncomplicated labor and vaginal delivery in a woman with COVID-19. The neonatal tested negative 72 hours after delivery. Our experience with this case indicated that there was no potential vertical transmission of the virus to the infant. This finding is in line with previous studies conducted in China [3-5]. A recent study showed that results of all samples (amniotic fluid, cord blood, neonatal throat swab, and breast milk) collected from nine live birth of COVID-19 infected mothers tested negative for SARS-CoV-2 [6]. Moreover, our patient's adherence to the launched recommendations by World Health Organization (WHO) on Breastfeeding for a COVID-19 confirmed mother [7] succeeded to avoid the spread of infection to the infant.

Our case report also described the negative impact of the COVID-19 on the mother mental health. Pregnant women may feel additional stress, anxiety, or depression during the COVID-19 pandemic. Hence, psychological interventions are needed to improve mental health of women during pregnancy and after delivery.

\section{Conclusions}

We reported a successful experience of safe vaginal delivery in a woman with COVID-19. As COVID-19 is rapidly spreading, maternal management and fetal safety remain a major concern. Obstetric hospitals should implement their emergency preparedness plan to ensure the safety of patients, clinicians and staff. In addition, health care worker should follow all updated infection control guidelines to keep themselves and others safe. Moreover, education of pregnant women about prevention of COVID-19 remains important strategy for protecting maternal and infant health. Breastfeeding is encouraged when all appropriate infection control measures are taken.

\section{Acknowledgements}

We would like to thank our patient for allowing us to publish her experience.

\section{Ethics Approval and Consent for Publication}

Single case reports are exempt from ethical approval in the Ministry of Public Health. Written informed consent was obtained from the patient for publication of this case report.

\section{Disclosure of Interest}

The authors report no conflict of interest.

\section{References}

1. Gao Q, Hu Y, Dai Z, et al. (2020) The epidemiological characteristics of 2019 novel coronavirus diseases (COVID-19) in Jingmen, Hubei, China. Med Rxiv 99: e20605.

2. Akalu $Y$, Ayelign B, Molla MD (2020) Knowledge, attitude and practice towards COVID-19 among chronic disease patients at Addis Zemen hospital, northwest Ethiopia. Infect Drug Resist 13: 1949-1960.

3. Yan J, Guo J, Fan C, et al. (2020) Coronavirus disease 2019 
(COVID-19) in pregnant women: A report based on 116 cases. Am J Obstet Gynecol 223: 111.e1-111.e14.

4. Chen Y, Peng H, Wang L, et al. (2020) Infants born to mothers with a new coronavirus (COVID-19). Frontiers in Pediatrics 8: 104.

5. Yang Z, Wang M, Zhu Z, et al. (2020) Coronavirus disease 2019 (COVID-19) and pregnancy: A systematic review. The Journal of Maternal-Fetal \& Neonatal Medicine 1: 1-4.
6. Chen H, Guo J, Wang C, et al. (2020) Clinical characteristics and intrauterine vertical transmission potential of COVID-19 infection in nine pregnant women: A retrospective review of medical records. Lancet 395: 809-815.

7. World Health Organization (WHO) (2020) Breastfeeding advice during the COVID-19 outbreak. 